\title{
Necrotizing Pneumonia: A Case Report on a Rare Complication of Respiratory Syncytial Virus Pneumonia \\ Rotana Hammad ${ }^{1}$, Khourshed Tewfik ${ }^{1}$, Shahinaz Munshi², Mohammed Felemban ${ }^{3}$ \\ Departments of ${ }^{1}$ Pediatric Pulmonary Medicine and ${ }^{2}$ Pediatric Allergy/Immunology, King Fahd Armed Forces Hospital, Jeddah, KSA \\ ${ }^{3}$ Jeddah Health Surveillance Centers, Ministry of Health, Jeddah, KSA
}

\begin{abstract}
Background: Necrotizing pneumonia is uncommon in children compared to adults. It is characterized by progressive pneumonic illness in a previously well child despite appropriate antibiotic treatment. This further leads to areas of necrosis and liquefaction of the consolidated lung tissue, which may be complicated by solitary, multiple, or multiloculated radiolucent foci, bronchopleural fistulas, and intrapulmonary abscess formation. Necrotizing pneumonia is usually secondary to Pneumococcus, Staphylococcus aureus, or, less commonly, Pseudomonas aeruginosa infections, and is rarely seen secondary to viruses. Although cavitary necrosis manifests as a severe disease, most children exhibit complete recovery even without surgical treatment and have normal chest radiographs in the long term.

Case report: In this report, a 3-year-old immunocompetent patient developed pleural effusion and empyema and underwent ultrasound-guided drainage without clinical improvement, leading to necrotizing pneumonia secondary to a respiratory syncytial virus and requiring video-assisted thoracoscopic surgery. We initiated conservative treatment with prolonged antibiotics, ventilator support, and analgesics.

Conclusion: Early surgical intervention in cases of necrotizing pneumonia is challenging, and it is unknown whether it is superior to medical management. Repeating chest radiographs should be considered in cases of changes in the patient's status or whenever there is a delay in recovery.
\end{abstract}

Keywords: Necrotizing pneumonia, Respiratory syncytial virus, Video-assisted thoracoscopic surgery

\section{INTRODUCTION}

Necrotizing pneumonia is a potentially fatal complication of pulmonary infection with high morbidity. Staphylococcus aureus strains that produce Panton-Valentine leucocidin, as well as Streptococcus pneumonia and Klebsiella pneumonia, are reportedly the most frequent cause of rapidly progressive necrosis of the lung tissue in young immunocompetent patients. Moreover, necrotizing pneumonia is less frequently secondary to fungi and viruses among immunocompetent patients. Fungi are more common in patients who are immunocompromised, causing cavitation and fibrotic changes in the lungs. Respiratory syncytial virus (RSV) is the most common cause of acute lower respiratory tract infections in infants and young children and has caused approximately 66,000 to 199,000 deaths among children worldwide in $2005^{(\mathbf{1})}$.

It is a single-stranded negative-sense RNA virus that belongs to the family of paramyxoviruses that is transmitted through respiratory droplets and contact. While the majority of infants display only mild upper respiratory tract infection, the virus can lead to bronchiolitis and severe lower respiratory tract infection in young children, and it is responsible for serious complications, respiratory failure, prolonged hospital stay, and even high mortality, especially in individuals who are immunocompromised. The typical histological finding of RSV pneumonia is the interstitial infiltration of mononuclear cells, which is mainly manifested as widening of the alveolar space and the predominant exudate interstitial monocyte ${ }^{(2)}$.

Here, we report a case of RSV pneumonia presenting as rapidly progressive necrotizing pneumonia in an immunocompetent patient.

\section{Case presentation:}

A previously healthy 3-year-old Saudi male presented to our Emergency Department, King Fahd Armed Forces Hospital, with fever, nasal congestion, and shortness of breath for 2 days. The patient had a history of coughing and had contact with his mother who has an upper respiratory tract infection. He was in respiratory distress, was tachypneic, had a respiratory rate of $44 \mathrm{bpm}$, was febrile with a temperature of $39^{\circ} \mathrm{C}$, had a desaturation of up to $88 \%$ on room air, and exhibited a fussiness with no cyanosis.

The initial laboratory findings revealed mild leukocytosis with a white blood cell count (WBC) of 10.3 $\mathrm{x} 10^{9} / \mathrm{L}$, neutrophil count of $57 \%$, lymphocyte count of $30 \%$, platelet count of $204 \times 10^{9} / \mathrm{L}$, and a C-reactive protein level of $166 \mathrm{mg} / \mathrm{L}$. The patient exhibited combined metabolic and respiratory alkalosis on blood gas and his blood culture showed no organism. 


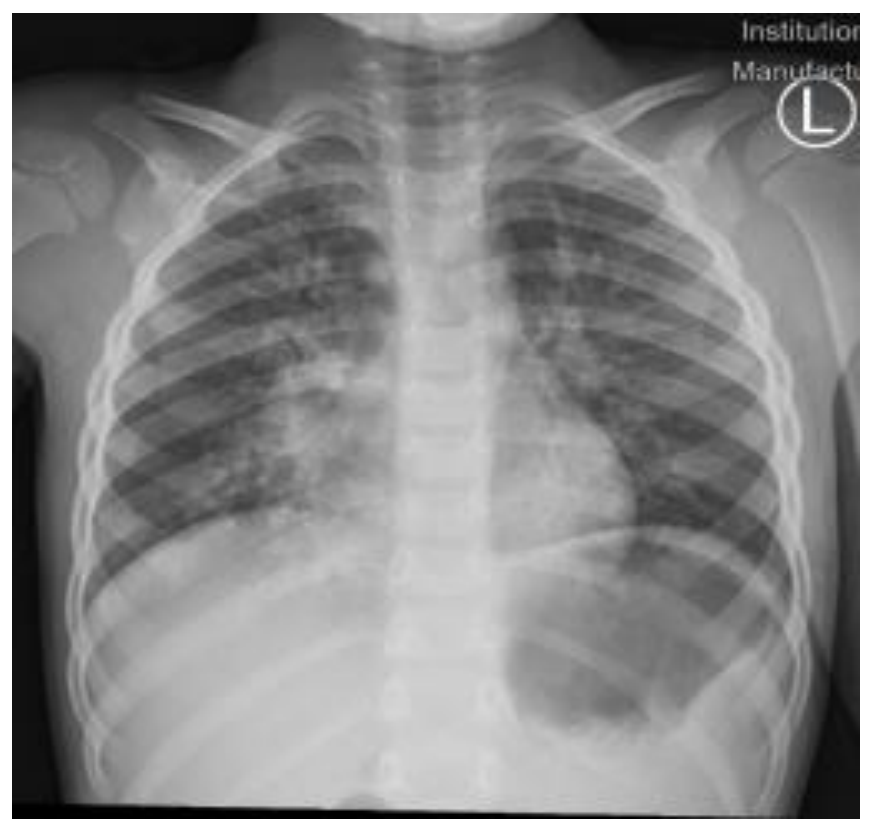

Fig. (1): Bilateral peripheral infiltration, right middle lobe infiltration and clear costophrenic angle bilaterally.

A complete viral respiratory multiplex panel revealed RSV with no other viruses involved. The initial chest radiograph showed bilateral peripheral infiltration and right middle lobe infiltration. However, the costophrenic angle was clear bilaterally (Fig. 1).

Conservative treatment was initiated in the form of good hydration and analgesics for fever. After 2 days the patient was transferred for a higher level of care in the pediatric intensive care unit because of an increase in oxygen requirement.

On admission, the patient was normotensive, tachypneic, had a respiratory rate of 60 , was distress, and had decreased oxygen saturation $(85 \%)$ on a 2 -L nasal cannula at a temperature of $40{ }^{\circ} \mathrm{C}$. On examination, the patient exhibited intercostal and subcostal retraction, crackles and decreased breath sounds over the lower zone of the left lung, and good perfusion.

His inflammatory markers increased, the (WBC) count was $23 \times 10^{9} / \mathrm{L}$, the neutrophil count was $72 \%$, the lymphocyte count was $11 \%$, the platelet count was $386 \mathrm{x}$ $10^{9} / \mathrm{L}$, the C-reactive protein level was $188 \mathrm{mg} / \mathrm{L}$, and the erythrocyte sedimentation rate was $83 \mathrm{~mm} / \mathrm{h}$. The coronavirus SARS-COV-2 repeat test was negative, as well as the methicillin-released $S$. aureus screening from the nose, axilla, and groin.

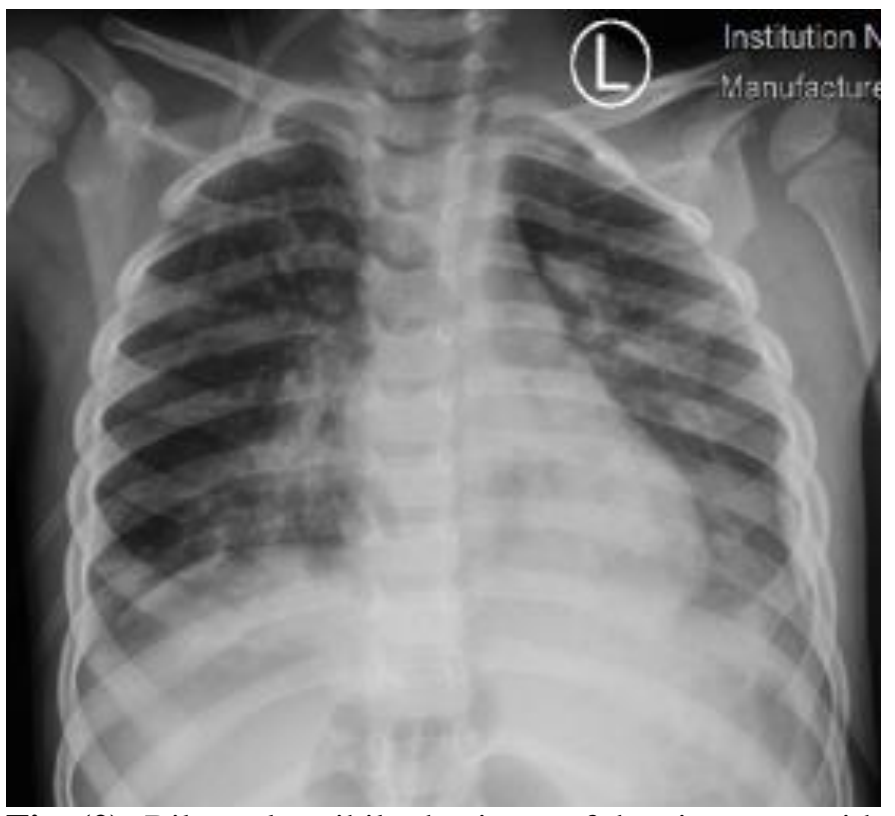

Fig. (2): Bilateral perihilar haziness of the air space, with areas of air bronchogram and bilateral obliteration of the costophrenic angle (more on the left side).

The chest radiograph in Fig. 2 shows bilateral perihilar haziness of the air space, with areas of air bronchogram and bilateral obliteration of the costophrenic angle (more on the left side).

This was confirmed by a chest ultrasound (US) (Fig. 3 ), which showed minimal bilateral simple free-flowing pleural effusion (more on the left side) with consolidation and no observed septations.

The echocardiogram was also normal. Respiratory support with bilevel positive airway pressure was initiated and was gradually weaned to a nasal cannula after 4 days. The patient was started on triple antibiotics (clindamycin, cefepime, and azithromycin) for 5 days.

Then, the antibiotics were changed to meropenem and vancomycin for broader spectrum coverage.

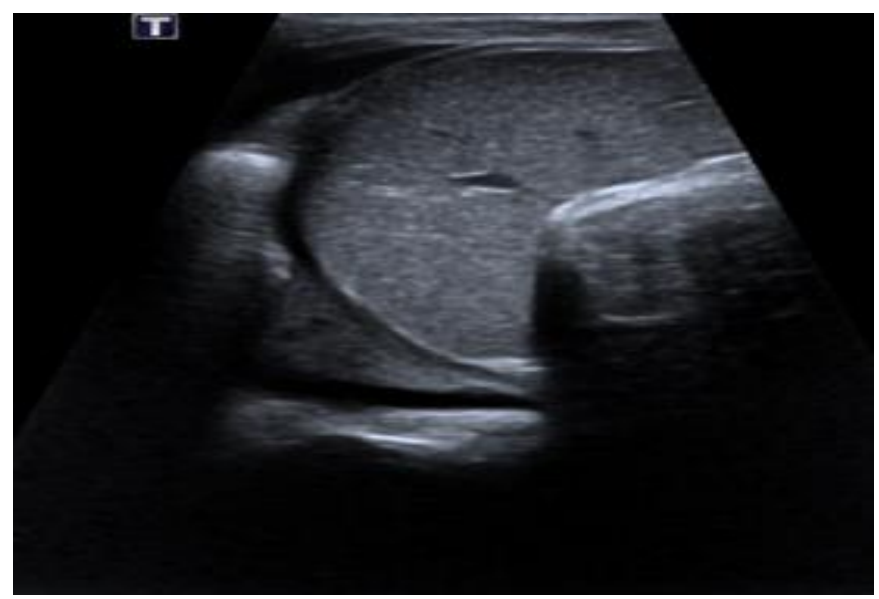

Fig. (3): Chest ultrasound (US) showed minimal bilateral simple free-flowing pleural effusion (more on the left side) with consolidation and no observed septations. 
The patient did not show much clinical improvement and was still tachypneic with spikes in temperature, hence we performed a chest computerized tomography (CT) scan (Fig. 4), which revealed mild bilateral pleural effusions (more on the left side), loculated with an enhancement of the left pleura.

These findings suggest parapneumonic effusions with early empyema formation. We also observed bilateral lung consolidations (more on the left lower lobe), with small necrotic parenchymal lung changes due to necrotizing pneumonia.

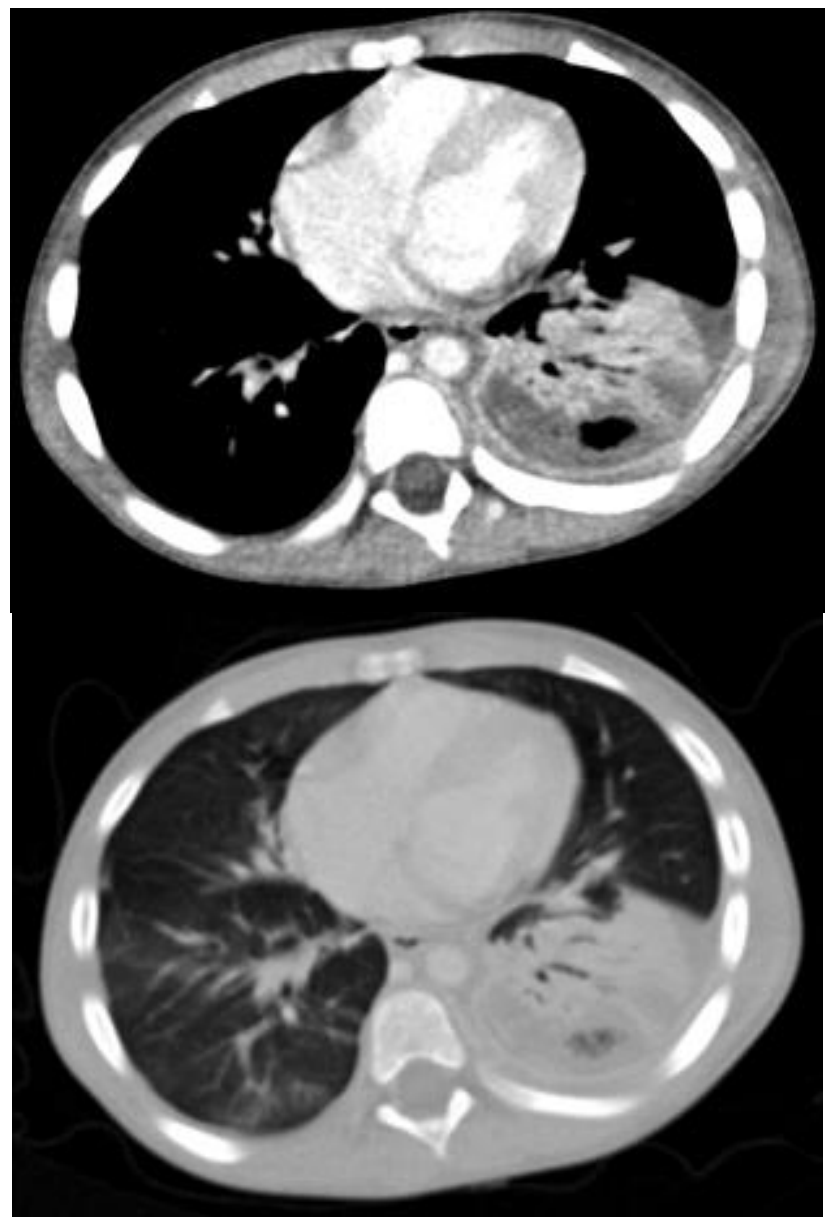

Fig. (4): Contrast-enhanced chest computerized tomography scan showed pneumonic consolidation with multiple areas of low attenuation suggestive of necrotizing pneumonia involving the entire left lower lobe.

We performed US-guided drainage of a minimal amount of blood-stained pleural fluid. We sent this fluid for cultures, and the findings were negative for any bacterial or fungal growth, as well as tuberculosis. Intravenous antibiotics were continued.

On the patient's $16^{\text {th }}$ day of admission, we performed video-assisted thoracoscopic surgery (VATS) for direct visualization of the pleural space in his left lung (Fig. 5) show adhesions of the pleura, areas of necrotic tissue in the pleural space, and a focal empyema that was excised.
The left lower lobe of the lungs was necrotic. Debridement was performed and the samples were sent for culture. A chest tube was placed and was removed after 2 days. The patient was maintained on room air with no complications apart from a daily spiking fever, which subsided after 5 days. The follow-up chest radiograph showed good expansion of the lung (Fig. 6).

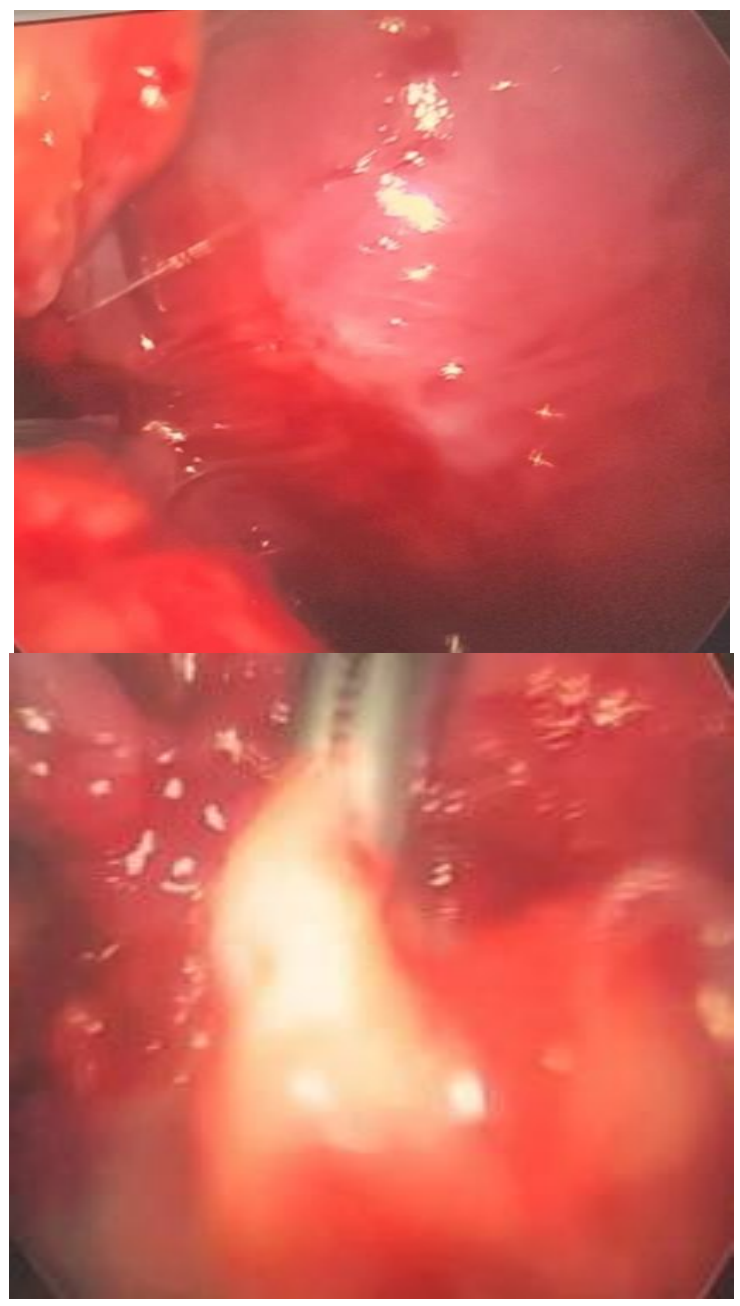

Fig. (5): Video-assisted thoracoscopic surgery showed areas of adhesions of the pleura, areas of necrotic tissue in the pleural space, and a focal empyema.

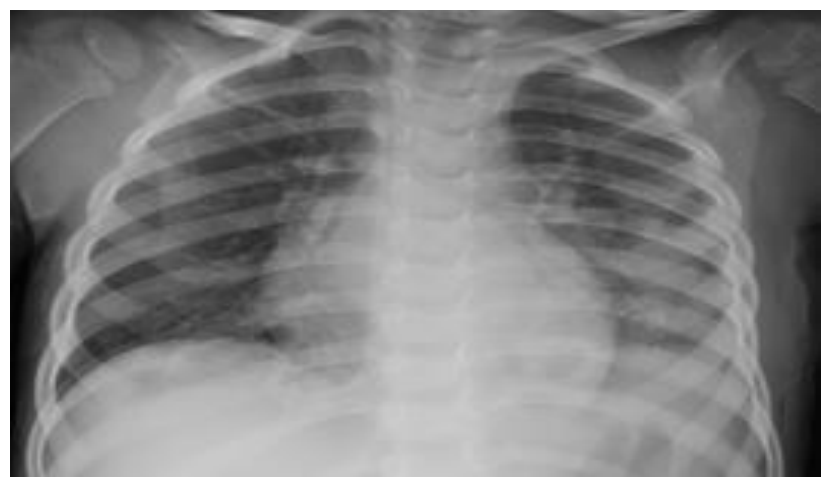

Fig. (6): Chest radiograph showed good expansion of the lungs.

Our patient continued on antibiotics treatment with clindamycin for 6 days, vancomycin for 24 days, and 
meropenem for 26 days. After 48 hours of being afebrile, he was shifted to oral third-generation cephalosporin to complete a total course of 6 weeks. During follow-up, the patient exhibited clinical and vital improvement. At the time of discharge, the patient was clinically and vitally stable as well. His chest examination on discharge exhibited clear bilateral air entry with good chest expansion. He was regularly followed up by a pediatric pulmonology team.

An approval of the study was obtained from King Fahd Armed Forces Hospital, Jeddah, Saudi Arabia Academic and Ethical Committee. The patient was informed that his case was took as case-report for publishing and he accepted. This work has been carried out in accordance with The Code of Ethics of the World Medical Association (Declaration of Helsinki) for studies involving humans.

\section{DISCUSSION}

Although necrotizing pneumonia is observed in various multiple lung infections, most cases described in the literature have been attributable to bacterial pathogens such as $S$. aurus, $\quad S$. pneumonia, and $K$. pneumoniae $^{(3)}$. Major epidemiological studies on the causative factors of necrotizing pneumonia revealed that preceding influenza infection is a very common feature ${ }^{(4-}$ 6). Because viral infection exhibits non-specific symptoms, it represents a high rate of suspected coinfection. Regarding host defense, pulmonary virus infections can reportedly impair the immune system and promote secondary pulmonary infections ${ }^{(7)}$. It is well known that the influenza virus is a strong proinflammatory stimulus in the lungs. The cytokine release causes an influx of immune cells such including neutrophils, monocytes and macrophages to the lung tissue (8, 9), which is essential during the immune responses to influenza pneumonia but will lead to an aggressive cytokine release known as a 'cytokine storm' that has been associated with influenza-related morbidity and mortality ${ }^{(\mathbf{1 0})}$.

The multiple interactions between pathogens and the host can be strictly strain-specific and probably differ between epidemics and geographical regions, which needs to be considered particularly for vaccine development ${ }^{(\mathbf{1 1}, \mathbf{1 2})}$.

In this report, we describe the case of a 3-year-old immunocompetent patient who presented with fever, nasal congestion, and shortness of breath. A high level of inflammatory markers was also observed, and the patient was found to have necrotizing pneumonia secondary to RSV. He needed to undergo surgical VATS for debridement of empyema and necrotic tissue in addition to a prolonged antibiotic course for the treatment of his condition. His symptoms were high-grade fever despite regular analgesic, crackles, and a lack of improvement after treatment with intravenous antibiotics.

Although viral infection with RSV is common in this pediatric age group, our patient's condition rapidly progressed in an atypical course of viral illness.

Our patient showed improvement on antibiotics combined with surgical intervention, raising the possibility of a superadded bacterial pathogen. However, the histopathological tissue culture and staining showed no growth of any additional organisms.

The mortality of necrotizing pneumonia is likely to be high, although it is difficult to assess because of its rarity. There is a lack of guidelines to direct the management of patients with necrotizing pneumonia, and surgical intervention versus combined surgical and medical management is needed.

\section{CONCLUSION}

Viral pneumonia requiring intensive care unit admission carries a high risk of morbidity, particularly when necrotizing pneumonia develops. The rapid deterioration of clinical conditions should highlight the importance of including the complications of pneumonia in an immunocompetent host. Radiological imaging such as chest CT is indicated for patients with a deteriorated clinical condition or pneumonia that is nonresponding to supportive measures and antibiotics. It will evaluate the parenchymal complications that are not appreciated on chest films and show any evidence of necrotizing pneumonia. Early surgical intervention in cases of necrotizing pneumonia is challenging, and it is unknown whether it is superior to medical management. Repeating chest radiographs should be considered in cases of changes in the patient's status or whenever there is a delay in recovery.

\section{Financial support and sponsorship: Nil. Conflict of interest: Nil.}

\section{REFERENCES}

1. Piedimonte G, Walton C, Samsell L (2013): Vertical transmission of respiratory syncytial virus modulates preand postnatal innervation and reactivity of rat airways. PLOS One, 8(4): 61309.

2. Wang Q, Li W, Qu D, Xin T et al. (2018): Fatal pulmonary infection with respiratory syncytial virus in an immunocompromised adult patient: A case report. Med. (Balti.), 97(29): 11528.

3. Tsai Y, Ku Y (2012): Necrotizing pneumonia: a rare complication of pneumonia requiring special consideration. Curr Opin Pulm Med., 18 (3): 246-252.

4. Gillet Y, Issartel B, Vanhems P et al. (2002): Association between Staphylococcus aureus strains carrying gene for Panton-Valentine leukocidin and highly lethal necrotising pneumonia in young immunocompetent patients. Lancet, 359(9308): 753-759. 
5. Gillet Y, Vanhems P, Lina G et al. (2007): Factors predicting mortality in necrotizing community-acquired pneumonia caused by Staphylococcus aureus containing Panton-Valentine leukocidin. Clin Infect Dis., 45(3): 315321.

6. Lina G, Piemont Y, Godail-Gamot $F$ et al. (1999): Involvement of Panton-Valentine leukocidin-producing Staphylococcus aureus in primary skin infections and pneumonia. Clin Infect Dis., 29(5): 1128-1132.

7. Löffler B, Niemann S, Ehrhardt $\mathrm{C}$ et al. (2013): Pathogenesis of staphylococcus aureus necrotizing pneumonia. Expert Rev Anti-Infect Ther., 11(10): 10411051.

8. Dessing M, van der Sluijs K, Florquin S et al. (2007): Monocyte chemoattractant protein 1 contributes to an adequate immune response in influenza pneumonia. Clin Immunol., 125(3): 328-336.
9. Wareing M, Lyon A, Lu B et al. (2004): Chemokine expression during the development and resolution of a pulmonary leukocyte response to influenza A virus infection in mice. J Leukoc Biol., 76(4): 886-895.

10. Bordon J, Aliberti S, Fernandez-Botran R et al. (2013): Understanding the roles of cytokines and neutrophil activity and neutrophil apoptosis in the protective versus deleterious inflammatory response in pneumonia. Int $\mathbf{J}$ Infect Dis., 17(2): 76-83.

11. Teijaro J, Walsh K, Cahalan S et al. (2011): Endothelial cells are central orchestrators of cytokine amplification during influenza virus infection. Cell, 146(6): 980-991.

12. McCullers J (2013): Do specific virus-bacteria pairings drive clinical outcomes of pneumonia? Clin Microbiol Infect., 19(2): 113-118. 